\title{
XXIII.
}

\section{Ueber die Hypertrophie und das physiologische Wachsthum des Herzens.}

\author{
Experimentelle und bistologische Untersuchung ans dem Institut für \\ allgemeine Pathologie und Therapie der Universität zu Budapest. \\ Von Dr. Franz Tangl, \\ II. Assistenten an pathologiseh-anatomischen Institut, \\ (Preisgekrönte Arbeit der Universität Budapest.)
}

Die genauen und sorgfältigen Untersuchungen Goldenborg's ${ }^{1}$ ) haben endgültig und vollkommen zweifellos die Thatsache festgestellt, dass die Muskelzellen hypertrophischer Herzen bedentend grösser sind als die normaler und dass die Hypertrophie wesentlich auf der Volumvergrösserung der Maskelzellen beruht. Goldenberg's Resultate stehen also in vollem Gegensatze zu denen Zielonko's ${ }^{2}$, zu welchen er sowohl bei experimentellen, als bei pathologisch-anatomischen Untersuchungen gelangte. (Auf die älteren und fast ausnahmslos nur auf wenigen Beobachtungen basirenden Literaturangaben will ich hier um so weniger reflectiren, als schon Goldenberg einen ziemlich vollständigen historischen Ueberblick über die Literatur der Herzhypertrophie gegeben hat.) Da Zielonko's Arbeit, soweit mir bekannt, die einzige experimentelle und histologische Untersuchung dieser Frage enthält, schien es mir nicht zwecklos, diese Frage experimentell wieder durchzaarbeiten, um so melır, da Zielonko's Experimente nur theilweise, an Kaltblütern, gelangen, an Kaninchen konnte er nur einmal eine Hypertrophie erzeugen, - und anderseits zu hoffen war, dass bei gïnstigen Experimenten vielleicht ein tieferer Einblick in den Zusammenhang zwischen der Grössenzunahme des Herzens und der Volumsvergrösserung

1) Goldenberg, Ueber Atroph. n. Hypertr. d. Muskelf. (l. Herzens. Dieses Archiv Bd. 103.

2) Zielonko, Path. anat. u. exp. Studien üb. Hypertr. d. Herzens. Dieses Archiv Bd. 62. S. 29. 
seiner Muskelelemente gewonnen werden kann. Auch glaubte ich auf diesem Wege eher und sicherer die eventuelle Vermehrung der Muskelzellen auffinden zu können. Im Folgenden theile ich die Resultate meiner Untersuchungen mit, bei deren Ausführung mir die Unterstützung des Herrn Professors Högyes zu Theil wurde, dem ich hier dafür meinen innigsten Dank ausspreche.

Vor Allem müssen die befolgten Methoden kurz angegeben werden, da deren Kenntniss zur Beurtheilung der Verlässlichkeit der gewonnenen Resultate bei dieser Frage besonders wichtig ist. Zur Erzeugung von Herzhypertrophie experimentirte ich nur an Kaninchen. Ich verfuhr dabei ganz nach den Angaben Rosenbach's's, indem ich mit einer, gewöhnlich durch die rechte Carotis eingefülurten Sonde eine oder zwei Klappen der Aorta durchbohrte oder zerstörte und so eine Aortenklappeninsufficienz hervorrief. Das Gelingen der Operation zeigten immer - wie es schon Rosenbach angiebt - die unmittelbar darnach auftretenden klinischen Symptome der Insufficienz. Nie fehlte das diastolische Geräusch über dem Herzen und der charakteristische Puls auch nicht. Oft war auch ein dentliches Schwirren wahrnehmbar. Da ich ausschliesslich nur diese Insufficienzoperation machte, entwickelte sich - wenn die nöthige Zeit dazu vorhanden war - nur die Hypertrophie des linken Ventrikels, was, wie es weiter ersichtlich sein wird, für die Untersuchung nur vortheilhaft war. Im Ganzen verfüge ich über 16 gelungene Experimente mit eingehender histologischer Untersuchung. Es soll gleich an dieser Stelle erwähnt sein, dass bei keinem meiner Experimente nach der Operation eine acute Endocarditis oder Endarteriitis sich entwickelt hat und dass die Thiere die Operation olne irgend welche entzündliche Reaction überstanden.

Besondere Sorgfalt musste auf die histologische Untersuchung und da auf die Art und Weise der mikroskopischen Messungen verwendet werden, da ich nur bei dem genauesten Vorgehen holfen durfte, meine Resultate mit denen Goldenberg's vergleichen zu können. Ich verfuhr folgendermaassen: Sowohl bei den normalen Herzen, die der Vergleichung wegen ebenso genau

1) Rosenbacb, Ueber artific. Herzfehler. Arch. f. exp. Pathol. Bd. IX. S.1. A rehiv fn pátıol. Anat. Bd, 116. Hft. 3. 
untersucht werden mussten, als bei den hypertrophischen, entnahm ich noch in ganz frischem Zustande immer derselbed Stelle der Wand des linken Ventrikels ein ganz kleines Stückchen mit der Scheere und legte es in 33 procentige Kalilauge $\left.{ }^{1}\right)$. Diese Lösung erhält - wie es schon Weismann, der sie zuerst zur Isolirung der Muskelzellen verwendete, angiebt -.. die Muskelzellen vorzüglich und löst nur die intercelluläre Kittsubstanz, ohne die Muskelzellen aufzulösen oder zu verändern. In der Kalilauge verblieb das Muskelstïckchen 30-45 Minuten und wurden ganz kleine Partikelchen desselben ebenfalls in Kalilauge auf den Objectträger gebracht, wo dieselben schon durch sanftes Schütteln mit der Präparirnadel in einzelne Zellen oder Zellgruppen zerfielen. Damit die Zellen durch das Deckglas nicht gequetscht werden, legte ich letzteres immer auf zwei Streifen dünnen sogenannten Seidenpapiers, so dass die Muskelzellen vor jedem Drucke geschützt waren. In einem so bereiteten Präparate nahm ich dann die Messung des Querdurchmessers der Muskelzellen vor und zwar so, dass ich denselben in jedem Falle an 100 Muskelzellen bestimmte, inmer mit demselben Ocularmikrometer Objectiv und Ocular und immer nur an solchen Zellen, die vollkommen unversehrt und gut isolirt waren. Nach 20 bis 30 Messungen erneute ich das Präparat. Aus 100 solchen Messungen berechnete ich dann das arithmetisehe Mittel, den Mittelwerth des Querdurchmessers. Ich glaube, das ist das einfachste und vielleicht auch sicherste Verfahren, da einerseits die frischen Muskelzellen der Wirkung nur eines Reagens ausgesetzt werden, welches sie kaum verändert, anderseits der Querdurchmesser mit kleinerem Fehler zu messen ist, als jn Schnitten, deren Richtung zur Längsaxe der Zellen nicht in jedem Falle ganz sicher bestimmt werden kann. Die Verlässlichkeit der Messungsresultate weiter zu prüfen, habe ich - durch Goldenberg's Beispiel angeregt - in einer bestimmten Anzahl von Fällen, nach den in Thoma's ${ }^{2}$ ) Buche angegebenen mathemati-

1) Der linke Ventrikel wurde immer am linken Rande eröfnet. Aus der Schnittfläche wurde dann etwa $2-3 \mathrm{~mm}$ von der Atrioventriculargrenze ein kleines Stückchen herausgeschnitten. Besonders achtete ich darauf, dass nicht etwa ein Theil des Papilarmuskels unter die Scbeere gerathe.

2) Thoma, Untersuchungen über die Grösse u. s. w. Leipzig 1882. 
schen Methoden in Mittelwerthe geprüft und die Resultate in entsprechenden Tabellen zusammengestellt.

Die in jedem Falle ausgeführte weitere histologische Untersuchung wurde an in Flemming'scher oder Müller'scher Flüssigkeit fixirten und gehärteten Präparaten angestellt. Meistens verwendete ich die erstere Lösung, die doch das verlässlichste Fixirungsmittel ist.

II.

Zum erfolgreichen Studium der Herzhypertrophie musste ich mir zuerst genaue Kenntnisse über die Grössenverhältnisse normaler Kaninchenherzen und deren histologischer Elemente verschaffen, und ausserdem das Wesen des physiologischen Wachsthumes des Herzens feststellen. Ich untersuchte also in erster Reihe normale Kaninchenherzen in dieser Beziehung.

Was die Grösse des Herzens betrifft, so kann diese durch drei Momente ausgedrückt werden: 1. durch das Volum des Herzens, bestimmt aus den Durchmessern desselben; 2. durch die Dicke der Herzwände - aus welcher man gewöhnlich auf Hypertrophie folgert -; 3. durch das Herzgewicht. Es frägt sich nun, welches von diesen dreien das verlässlichste Maass дег Grösse des Herzens giebt, was besonders bei der Feststellung und Abschätzung der Herzhypertrophie von Wichtigkeit ist. Alle drei sind selbst bei gleich grossen Thieren beträchtlichen individuellen Schwankungen unterworfen, doch hat ausserdem die Bestimmung der Durchmesser des Herzens und die Dicke der Herzwände noch eine sehr in Betracht kommende Fehlerquelle. Man denke nur, wie diese zwei Momente in ihrem Werthe variiren, je nachdem das Herz in Systole oder.Diastole stehen geblieben ist. Den diesem Umstande entspringenden Fehler können wir ganz sicher bei der Bestimmung des Herzgewichtes ausschliessen, da die Contractionsphasen den Werth desselben. nicht beeinflussen. Ich habe deshalb zur Grössenbestimmung des Herzens das Gewicht desselben gewählt - natürlich mit den nöthigen Cautelen - um so mehr als auch Thoma angiebt, dass es ihm gelungen ist aus dem Herzgewichte, bezw. aus dem relativen Herzgewichte, kleine Hypertrophien zu erkennen; was ihm auf anderen Wegen kaüm gelungen wäre. 
Es wurde also bei normalen Kaninchen das Herzgewicht und dessen Verhältniss zum Körpergewicht bestimmt und festgestellt innerhalb welcher Grenzen diese Werthe variiren. Ich habe 50 solcher Gewichtsbestimmungen gemacht. Das Verhältniss zwischen Herzgewicht ${ }^{1}$ ) und Körpergewicht ist so ermittelt worden, dass berechnet wurde, wie viel Herzgewicht auf $1000 \mathrm{~g}$ Körpergewicht fällt. Diese Verhältnisszahl will ich weiterhin mit $R=$ relatives Herzgewicht bezeichnen. Ich wählte kleinere und grössere Thiere, so wie ich sie auch zu den Experimenten genommen habe. Der aus diesen 50 Messungen gewonnene Mittelwerth des relativen Herzgewichtes ist $257,9 \mathrm{cg}$; der kleinste beobachtete Werth des $\mathrm{R}$ war $200 \mathrm{cg}$ der grösste $314 \mathrm{cg}$. Abgesehen von sehr jungen Individuen - die natürlich nicht unter die 50 Fälle aufgenommen sind, - zeigte $R$ keinen wesentlichen Unterschied bei grösseren und kleineren Thieren. Die ziemlich grosse Zahl der Fälle, aus denen der Mittelwerth des $\mathrm{R}$ bestimmt wurde, bot schon an und für sich genügende Garantie für die Verlässlichkeit dieses Werthes. Die mathematische Prüfung desselben erwies dasselbe; der wahrscheinliche Werth des Bestimmungsfehlers dieser Mittelzahl beträgt nur $2,7 \mathrm{cg}$, was also nur $1,04 \mathrm{pCt}$. derselben ausmacht. $257,9 \mathrm{cg}$ kann also als normales relatives Herzgewicht gelten.

Ausser $\mathrm{R}$ wurde noch bei 28 der oben angeführten $50 \mathrm{Be}$ obachtungen das Gewichtsverhältniss zwischen Herz und Nieren bestimmt, da nach Grawitz und Israel ${ }^{2}$ ) dieses Verhältniss bei Kaninchen viel beständiger sein soll, als dasjenige zwischen Herz- und Körpergewicht. Sie fanden bei normalen Herz und Nieren : $\frac{\text { rechte Niere+linke Niere }}{\text { Herzgewicht }}=$ I (Index) immer grösser als 2,5. Aus meinen 28 Fällen habe ich als Mittelwerth für I 2,82 erhalten. I schwank jedoch innerhalb ebenso weiter Grenzen als $R$, und der wahrscheinliche Werth des Bestimmungsfehlers seines Mittelwerthes beträgt 2,12pCt. desselben. I ist also an und

) Herzgewicht $=$ Ventrikel + Vorhöfe + Wurzel der grossen Gefässe. Vor dem Wägen wurde das Herz sorgfältig mit Wasser gereinigt and abgetrocknet und darauf geachtet, dass die grossen Gefässe immer in gleicher Höhe abgeschnitten wurden.

$\Rightarrow$ Grawitz u. Israel, Dieses Archiv Bd. 77. S. 315. 
für sich nicht constanter als $R$, beide zusammen liefern aber ein verlässlicheres Maass für die Grösse des Herzens, als jedes einzeln.

Tabelle I bietet einen Ueberblick der besprochenen Gewichtsverhältnisse, die sich hier auf 10 Beobachtungsreihen beziehen.

\section{Tabelle I.}

Gewichtsverhälnisse normaler Kaninchenherzen.

\begin{tabular}{r|c|c|c|c}
\hline No. & Köpergewicht & $\begin{array}{c}\text { Abs. } \\
\text { Herzgewicht }\end{array}$ & $\begin{array}{c}\text { R= relat. Herz- } \\
\text { gewichtauf 1000g } \\
\text { Körpergewicht } \\
\text { berechnet }\end{array}$ & $I=\frac{\text { r. Niere+l. Niere }}{\text { Herz }}$ \\
\hline 1 & $610 \mathrm{~g}$ & $181 \mathrm{cg}$ & $297 \mathrm{eg}$ & 2,98 \\
2 & $950-$ & $249-$ & $262-$ & 2,69 \\
3 & $980-$ & $304-$ & $310-$ & 2,41 \\
4 & $1000-$ & $233-$ & $233-$ & 2,98 \\
5 & $1150-$ & $333-$ & $289-$ & 2,23 \\
6 & $1270-$ & $339-$ & $267-$ & 2,85 \\
7 & $1340-$ & $309-$ & $231-$ & 3,31 \\
8 & $1410-$ & $429-$ & $304-$ & 2,56 \\
9 & $2300-$ & $549-$ & $239-$ & 2,96 \\
10 & $2600-$ & $641-$ & $247-$ & 2,15
\end{tabular}

Diese Tabelle zeigt zugleich die Grösse des absoluten Gewichtes normaler Kauinchenherzen, und kann später bei der Besprechung der Hypertrophie zur Vergleichung dienen. Wie es auch schon aus diesen wenigen Daten ersichtlich ist, wächst mit dem Körpergewicht auch das absolute Herzgewicht. Bei den kleineren Thieren variirte letzteres zwischen $240-300 \mathrm{cg}$, bei grösseren zwischen $400-600 \mathrm{cg}$. Das grösste normale Kaninchenherz von eincm $3200 \mathrm{~g}$ schweren Thiere wog $640 \mathrm{cg}$.

Was die histologischen Veränderungen betrifft, auf welchen das physiologische Wachsthum des Herzens beruht, sind die Ansichten der Forscher getheilt. Nach Goldenberg und Letulle jst die Volumvergrösserung der Muskelzellen das Wesentliche; die numerische Vermehrung ist nur nebensächlich. Langerhans ${ }^{1}$ ) fand die Herzmuskelzellen neugeborner und erwachsener Kaninchen gleich gross; dasselbe fand er auch beim Menschen. Eberth ${ }^{2}$ ) sah dasselbe und glaubt deshalb auch, dass die Zellen

1) Langerbans, Dieses Archiv Bd. 58. S. 65.

2) Ebertb, Dieses Archiv Bd. 37. S. 100. 
sich nicht vergrössern, sondern nur vermehren. Meine Untersuchungen über die Grösse der Muskelzellen beschränken sich nur auf die Wand des linken Ventrikels, da es festgestellt ist, dass in den verschiedenen Theilen des Herzens dieselben Veränderungen stattfinden und anderseits mit Rücksicht auf meine Hypertrophieexperimente nur der linke Ventrikel in Betracht kam.

Ich habe nach der oben angegebenen Methode an 16 normalen Kaninchenherzen den Querdurchmesser der Muskelzellen des linken Ventrikels bestimmt, indem ich jn jedem Falle aus je 100 Einzelmessungen den Mittelwerth des Querdurchmessers (D) berechnete. Die Resultate dieser Bestimmungen sind iu Tabelle II zusammengestellt.

\section{Tabelle II.}

Muskelzellenquerdurchmesser normaler Kaninchenherzen.

$D=$ Mittelwerth des Querdurchmessers aus je 100 Messungen.

\begin{tabular}{c|c|c|c|c|c}
\hline No. & Abs. Herzgewicht & $\mathrm{D}$ & No. & Abs. Herzgewicht & $\mathrm{D}$ \\
\hline 1 & $21 \mathrm{cg}$ & $5,57 \mu$ & 9 & $304 \mathrm{cg}$ & $14,21 \mu$ \\
2 & $93-$ & $8,44-$ & 10 & $309-$ & $13,23-$ \\
3 & $169-$ & $10,18-$ & 11 & $333-$ & $13,84-$ \\
4 & $247-$ & $13,01-$ & 12 & $350-$ & $15,43-$ \\
5 & $254-$ & $12,84-$ & 13 & $429-$ & $14,92-$ \\
6 & $265-$ & $11,21-$ & 14 & $460-$ & $15,40-$ \\
7 & $266-$ & $12,70-$ & 15 & $539-$ & $15,67-$ \\
8 & $294-$ & $11,44-$ & 16 & $624-$ & $15,33-$
\end{tabular}

Die Herzen sind hier der Grösse nach aufgezählt. Nr. 1 ist das eines neugebornen, Nr. 2 das eines einige Wochen alten Kaninchens: Bezüglich der anderen gilt es im Allgemeinen, dass je grösser das Herz, desto grösser das Thier, was bis zu einer gewissen Grenze mit dem Alter desselben zusammenhängt. - Ein Blick auf die Mittelwerthe der Muskelzellendurchmesser genügt sofort den grossen Unterschied desselben bei verschieden grossen Herzen zu zeigen. D ist bei Kaninchen Nr. 16 fast dreimal so gross wie beim neugebornen Thiere. Aus der Tabelle ist auch ersichtlich, dass durchschnittlich je grösser das Herz, desto grösser der Mittelworth des Zellenquerdurchmessers, also desto dicker die Muskelzellen. Noch deutlicher wird dieses Verhältniss, wenn wir die Herzen, die der Grösse nach von einander nur wenig differiren, in eine Gruppe zu zusammenfassen und aus 
den D dieser Gruppe eine Gesammtmittelzahl berechnen. Solche Gruppen mit der Gesammtmittelzahl sind in Tabelle III gebildet, welche zugleich die Daten der Tabelle II graphisch darstellt.

\section{Tabelle III.}

Vergrösserung der Muskelzellenquerdurchmesser beim physiologischen Wachsthume des Herzens.

$\mathrm{D}=$ Mittelwerth des Querdurchmessers aus je 100 Messungen bestimmt.

$D^{\prime}=$ Gesammtmittelwerth einer Gruppe aus den D derselben berechnet.

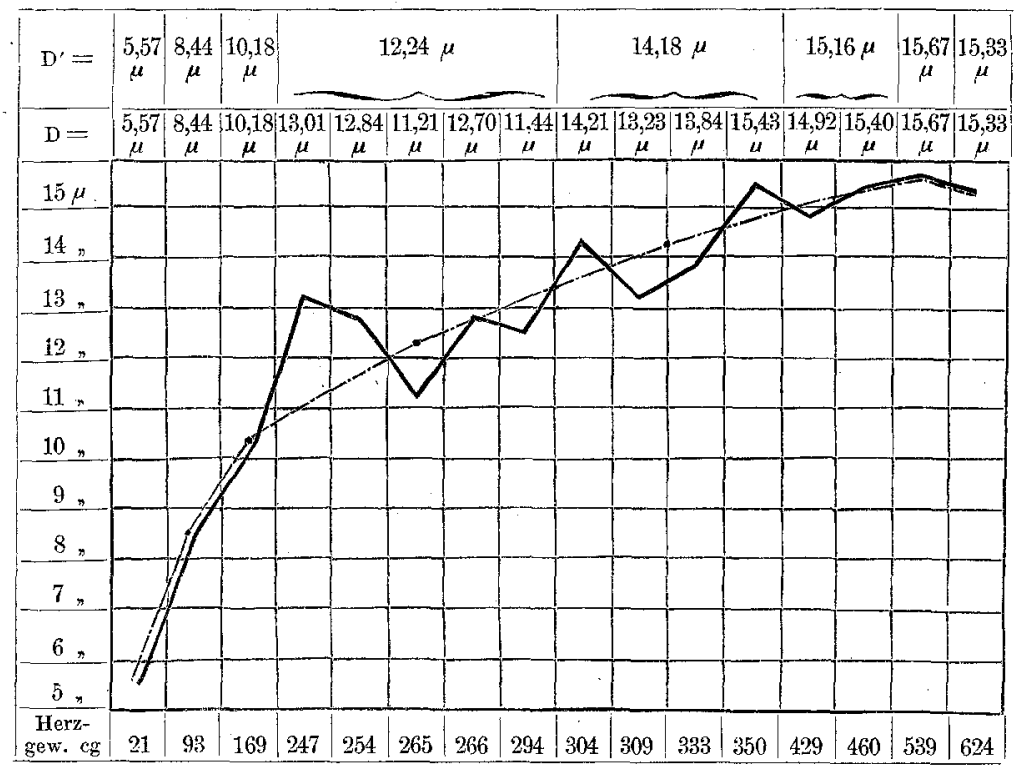

Die Herzen sind ihrer Gewichtsgrösse nach in der Abscissenaxe aneinander gereiht, die entsprechenden Mittelwerthe der Muskelzellenquerdurchmesser (D) auf die Ordinatenaxe aufgetragen. In einer Gruppe beträgt die Differenz zwischen dem kleinsten und grössten Herzgewicht nicht mehr als $50 \mathrm{cg}$. Nach diesem Principe sind die Gruppen gebildet und für jede ist ein Gesammtmittelwerth der Muskelzellendurchmesser berechnet. (Oberste horizontale Reihe der Tabelle III.) Die ausgezogene Curve stellt die Veränderung von D, die punctirte die des Gesammtmittelwerthes dar. Beide Curven, besonders aber die punctirte der Gesammtmittelwerthe, drücken das Verbältniss zwischen Herzgewicht und Muskelzellenquerdurchmesser aus und versinnlichen 
die Thatsache, dass beim physiologischen Wachsthume des Herzens mit dem Gewichte desselben auch die Querdurchmesser der Muskelzellen grösser werden.

Diese Folgerung kann nur dann richtig sein, wenn der Mittelwerth des Querdurchmesser der Muskelzellen ein verlässliches Maass für die Breite der Muskelzellen ist, wenn derselbe also den Anforderungen der Wahrscheinlichkeitsrechnung entspricht. Das musste nach mathematischen Methoden geprüft werden. Ich berechnete also - so wie Goldenberg - nach der Methode der kleinsten Quadrate für 10 Beobachtungsreihen: $\boldsymbol{W}=$ den wahrscheinlichen Werth der Abweichung der einzelnen Messungen vom Mittelwerthe $\mathrm{D} ; \mathrm{F}=$ den wahrscheinlichen Werth des Bestimmungsfehlers der Mittelzahl D. - Diese Resultate sind im 1. Theil der folgenden Tabelle IV zusammengefasst.

Tabelle IV.

D = Nittelwerth des Querdurchmessers der Muskelzellen aus je 100 Messungen bestimmt;

$\mathrm{F}=$ wahrscheinlicher Werth des Bestimmungstehlers der Mittelzahl $\mathrm{D}$;

$W=$ wahrscheinlicher Werth der Abweichung der einzelnen Messungen von der Mittelzahl D.

(Im Theil 2.: Häufigkeit und Grössenwerth der einzelnen Abweichungen von den Mittelwerthen D.)

\begin{tabular}{|c|c|c|c|c|c|c|c|c|c|c|c|c|c|c|c|}
\hline \multicolumn{5}{|c|}{1.} & \multicolumn{11}{|c|}{2.} \\
\hline No. & $\begin{array}{c}\text { Abs. } \\
\text { Herz- } \\
\text { gewieht }\end{array}$ & $\begin{array}{l}\mathrm{D} \\
\mu\end{array}$ & $\begin{array}{l}\text { W } \\
\mu\end{array}$ & $\begin{array}{l}F \\
\mu\end{array}$ & 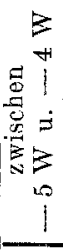 & 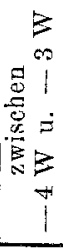 & 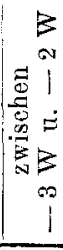 & 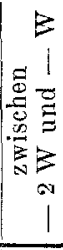 & 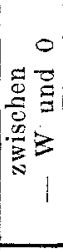 & 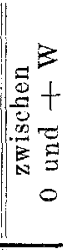 & 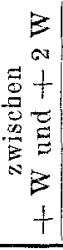 & 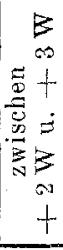 & 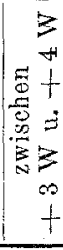 & 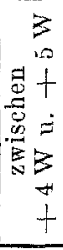 & $\begin{array}{r}2 \\
0 \\
5 \\
0 \\
0 \\
0 \\
0 \\
0 \\
0 \\
0 \\
0 \\
1\end{array}$ \\
\hline 4 & $47 \mathrm{cg}$ & 01 & 1,95 & 8 & 0 & 4 & 1 & 12 & 37 & 23 & 16 & .3 & 4 & 0 & 0 \\
\hline 5 & $254-$ & 1 & 2,01 & 0 & 0 & 1 & 6 & 14 & 32 & 29 & 13 & 9 & 1 & 0 & 0 \\
\hline 7 & $266-$ & 12,7 & 1,7 & 0 & 0 & 1 & 4 & 11 & 41 & 26 & 13 & 0 & 3 & 1 & 0 \\
\hline 9 & $304-$ & & 1,7 & 0,1 & 0 & 0 & 7 & 24 & 33 & 24 & 10 & 2 & 0 & 0 & 0 \\
\hline 10 & 309 & & 1,6 & & 0 & 3 & 5 & 2 & 34 & 36 & 14 & 1. & 4 & 0 & 1 \\
\hline 12 & $350-$ & 15 & 2,28 & 0,2 & 0 & 1 & 6 & 12 & 31 & 30 & 10 & 5 & 3 & 2 & 0 \\
\hline 13 & $429-$ & 14,92 & 1,68 & 0,17 & 0 & 1 & 0 & 19 & 32 & 33 & 10 & 1 & 4 & 0 & 0 \\
\hline 14 & 460 & 15,40 & 2,1 & 0,2 & 0 & 0 & 3 & 11 & 43 & 27 & 8 & 3 & 2 & 2 & 1 \\
\hline 15 & & 15,6 & 1,9 & 0,1 & 0 & 2 & 14 & 1 & 27 & 34 & 14 & 6 & 2 & 0 & 0 \\
\hline 16 & $624-$ & 15,33 & 2,15 & 0,22 & 0 & 1 & 6 & 14 & 30 & 25 & 16 & 4 & 4 & 0 & 0 \\
\hline \multirow{2}{*}{\multicolumn{5}{|c|}{$\begin{array}{l}\text { Die } 1000 \text { Messungen summirt } \\
\text { Die Theorje verl.f. } 1000 \text { Jessung. }\end{array}$}} & 0 & 14 & 52 & 120 & 340 & 282 & $1 У 4$ & 34 & 27 & 7 & 2 \\
\hline & & & & & 4 & 18 & 67 & 161 & 250 & 250 & 161 & 67 & 18 & 4 & 0 \\
\hline - & - & - & 100 & & 1 & 2 & 6 & 16 & 25 & 25 & 16 & 6 & 2 & 1 & - \\
\hline
\end{tabular}


Im 2. Theil dieser Tabelle sind die Häufigkeitszahlen der Abweichung der einzelnen Messungen von der Mittelzahl nach vielfachen der Zahl W zusammengestellt. Der Bestimmungsfehler liegt also nach dieser Tabelle weit innerhalb der erlaubten Grenzen und auch die Häufiglseitszahlen entsprechen im Allgemeinen der Theorie der Wahrscheinlichkeitsrechnung. Doch sind in der Reihe der summirten 1000 Abweichungen Asymmetrien wahrnehmbar, die fast ganz mit denen übereinstimmen, die Goldenberg bei seimen Messungen fand und welche ich bei den hypertrophischen Herzen auch gefunden habe.

Goldenberg giebt an der entsprechenden Stelle ${ }^{1}$ ) seiner Arbeit nach den diesbezüglichen Ausführungen Thoma's eine eingehende Erklärung dieser Erscheinung. Eine Erscheinung kommt aber in meinen Messungsreihen vor, die sich in denen Goldenberg's nicht vorfand und die eine specielle Erwähnung verdient. In zwei Beobachtungsreiben kommt nehmlich je eine Abweichung vor, die zwischen $+5 \mathrm{~W}$ und $+6 \mathrm{~W}$ liegt, während die von der Theorie erlaubte Grenze $5 \mathrm{~W}$ ist. Bezüglich dieser Erscheinung ist die Frage berechtigt: soll die entsprechende übermässig dicke Muskelzelle zur Bestimmung der Mittelzahl herangezogen oder aus der Berechnung ausgeschlossen werden? Die Lösnng dieser Frage verdanke ich einer frenndlichen brieflichen Mittheilung von Herrn Professor Thoma. Diejenige Zelle ist aus der Berechnung nicht auszuschliessen, weil die durch Weglassung derselben bedingte Veränderung des Mittelwerthes noch weit innerbalb der Grenze des Bestimmungsfehlers liegt und wir anderseits absolut keinen hinreichenden Grund haben, diese Zelle auszuschliessen, da dieselbe von den anderen histologisch gar nicht verschieden ist. Alle die erwähnten Asymmetrien und Unregelmässigkeiten in den Messungsreihen sind nach Thom a darauf zurückzufüren, dass die Muskelzellen kein "homogenes Material" der Beobachtung bilden, d. h. die Muskelzellen sind nicht alle gleichwerthig, weil sie nicht auf gleicher Stufe der Entwickelung stehen; bei den Messungen haben wir aber keine Anhaltspunkte diese ungleich entwickelten Zellen von einander zu unterscheiden. (Man muss ungleichwerthige Muskelzellen auch dann noch annehmen, wenn man auch mit Golden1) Goldenberg, a. a. O. S. 101. 
berg 's Eintheilung in Bündelfasern und Kettenfasern nicht einverstanden ist.)

Die mitgetheilten Messungsresultate zeigen also ganz sicher, dass beim Wachsen des Herzens die Muskelzellen in die Breite wachsen, dass sie dicker worden, sie lassen aber keineswegs folgern, dass sie sich auch verlängern. Dass die Muskelzellen sich in der Richtung ihrer Längsaxe auch vergrössern, davon konnte ich mich überzeugen, obzwar ich diesbezüglich keine methodischen Messungen angestellt habe. Die Herzmuskelzellen neugeborner Kaninchen sind bedeutend kürzer als die erwachsener, und in kleinen Herzen sind sie im Allgemeinen kürzer als in grösseren. Noch cin Befund weist auf das Längenwachsthum der Zellen hin. In Längsschnitten junger Herzen findet man die Muskelkerne viel dichter hinter einander liegen, als in entwickelteren Herzen, was - da die Zellen nur ein- oder höchstens zweikernig sind - nur so erklärlich ist, dass die Kerne durch das Längerwerden der Zellen weiter von einander gerathen. Die Muskelzellen vergrössern sich also beim Wachsen des Herzens in allen drei Dimensionen.

Die nächste Frage ist die, ob neben der Volumszunahme die Muskelzellen sich auch vermehren? Was ich zur Lösung dieser nicht leichten Frage beitragen kann, ist Folgendes: Es ist sicher, dass sich die Muskelzellen im embryonalen und neugebornen Herzen durch Theilung vermehren. Ich kann das deshalb behaupten, da ich in den erwähnten Herzen in den Maskelzellen ziemlich viel Mitosen fand und die Zellen selbst sich bei der Isolirung mit $\mathrm{KOH}$ grösstentheils als einkernige, in geringer Zahl als zweikernige erwiesen. Es mïssen sich also die Zellen getheilt haben. Ueber den Theilungsmodus kann ich nichts Bestimmtes sagen. Es soheint die Theilung in der Länge der Zellen zu geschehen, dafür spräche auch die Spindelform der Zellen zu jener Zeit. Die Zellen liegen dicht aneinander and sind durch foine Seitenäste mit einander schon verbunden. Bis zu welcher Zeit die Theilung der Muskelkerne und mit ihr die der Zellen anhält, konnte ich nicht feststellen, da meine diesbezüglichen Beobachtungen lückenhaft sind. Im $93 \mathrm{cg}$ schweren Herzen eines einige Wochen alten Kaninchens konnte ich keine Mitosen mehr finden; auch war die Gestalt der Muskelzellen 
nicht mehr spindelförmig, sondern den Zellen entwickelter Herzen ähnlich. Es wäre jedoch nicht unmöglich, dass selbst nach dem Aufhören der Kerntheilung die zweikernigen Zellen sich noch später theilten. Was die Erscheirungen betrifft, die in entwickelteren Herzen auf Theilung der Múskelzellen hinweisen, darauf will ich bei Besprechung der hypertrophischen Herzen näher eingehen. Hier sei nur erwähnt, dass man zwar diese Theilung, und so die numerische Vermehrung der Muskelzellen nicht sicher ausschliessen kann, aber auch kein sicheres Zeichen dafür findet. Jedenfalls spielt aber diese eventuelle Vermehrung nur eine sehr nebensüchliche Rolle beim Wachsthum des Herzens neben der Volumsvergrösserung der Zellen. Approximative Berechnungen zeigen auch, dass, vorausgesetzt, dass die Zellen sich in allen Dimensionen in gleichem Verhältnisse vergrössern, die einfache Volumsvergrösserung der Muskelzellen ohne Vermehrung und abgesehen von den Veränderungen der übrigen histologischen Bestandtheile, vollkommen genügen würde die thatsächliche Gewichtszunahme des Herzens und so dessen Wachsthum zu erklären.

Ich kann also das über das physiologische Wachsthum des Herzens Gesagte in Folgendem zusammenfassen:

Im embryonalen Leben und eine Zeit lang nach der Geburt wächst das Herz durch Volumszunahme und Theilung der Muskelzellen. Später berubt das Wachsthum des Herzens wesentlich auf der Vergrösserung der Muskelzellen.

\section{III.}

Dies vorausgeschickt, kann ich zur Besprechung der hypertrophischen Herzen übergehen. Wie ich die Herzhypertrophie an Kaninchen erzeugte, habe ich schon Eingangs angegeben (S. 433). Im Ganzen wurden 16 Kaninchenherzen mit Aortenklappeninsufficienz untersucht. Auch wurde schon erwähnt, dass sich bei keinem meiner Experimente eine acute Endocarditis. oder Endarteriitis entwickelte. Die Verletzung, die meistens nur eine Klappe traf, bestand gewöhnlich nur darin, dass auf der Klappe eine mit ihrer Basis mehr oder minder parallele Spalte entstand, deren Länge einigemal fast die Breite der Klappe er- 
reichte. Die spalte blieb weiterhin klaffend und wurde dann bei der Section meistens mit glatten, kaum verdickten Rändern gefunden. In ein oder zwei Fällen wurde die Klappe bei der Operation dermaassen zerstört, dass ich bei der Section kaum ihre Spuren gefunden habe, so sehr war sie zusammengeschrumpft. In zwei Fällen entstand auf der verletzten Klappe keine Spalte, sondern nur ein rundes Loch, welches in einem Falle noch dadurch verkleinert wurde, dass sein Rand verdickt war (126 Tage nach der Operation).

Die erste morphologische Veränderung, welche das Herz nach dem durch die Verletzung bedingten Auftreten der Aortenklappeninsufficienz erleidet, ist die Dilatation des linken Ventrikels. Ich kann also Rosenbach's Behauptung bestätigen, dass bei Aortenklappeninsufficienz der Hypertrophie des linken Ventrikels ausnahmslos dessen Dilatation vorausgeht. Die Dilatation entspricht der Grösse des Klappendefectes, was selbstverständlich ist, da bei grösserem Defecte während der Diastole mehr Blut regurgitirt und der Druck auf dio Ventrikelwand ein grösserer wird. Die Dilatation scheint auch dann noch fortzuschreiten, wenn die Ventrikelwand schon hypertrophisch geworden ist; wenigstens weist der Umstand darauf hin, dass selbst bei hochgradiger Hypertrophie die Dilatation so bedentend war, dass die Ventrikelwand gar nicht oder kium verdickt erschien. Da das Her\% nicht sofort nach Eintritt der Klappeninsufficienz hypertrophirt, sondern sich nur erweitert, muss man annehmen, dass dessen Musculatur über gewisse Reservekräfte (Rosenbach) verfügt, mit deren Hülfe sie die vermehrte Arbeitsleistung zu bewältigen fähig ist. Nach einer gowissen Zeit fängt jedoch die Muskelmasse an sich zu vermehren; die Musculatur hypertrophirt. Wann die Hypertropbie anfängt, hängt ausser vom Grade der Insufficienz noch wahrscheinlich von dem Ernährungszustande des Herzens ab. Zuerst habe ich arn 19. Tage der Insufficienz die Hypertrophie des linken Ventrikels nachweisen können. Ueber diese Zeit hinaus war Hypertrophie immer vorhanden. (Siehe Tab. V.) In zwei Fällen, einmal am 11. Tage und einmal am 12. Tage des Bestehens der Insufficienz, habe ich nur Dilatation und noch keine Hypertrophie gefunden. 
Tabelle V.

Kaninchenherzen mit känstlicher Aortenklappeninsufficienz.

\begin{tabular}{|c|c|c|c|c|c|c|}
\hline No. & $\begin{array}{l}\text { Kör- } \\
\text { per- } \\
\text { gew. }\end{array}$ & $\begin{array}{l}\text { Abs. } \\
\text { Herz- } \\
\text { gew. }\end{array}$ & $\mid \begin{array}{c}\mathrm{R}=\text { rel. } \\
\text { Herzgew. } \\
\text { auf } 1000 \mathrm{~g} \\
\text { Körper- } \\
\text { gewicht } \\
\text { berechnet }\end{array}$ & $\begin{array}{c}J==1 . \text { Niere } \\
+ \text { r. Niere } \\
\text { Herz }\end{array}$ & $\begin{array}{c}\text { Zeit- } \\
\text { dauer } \\
\text { der } \\
\text { Insuf- } \\
\text { ficient }\end{array}$ & Sectionsbefund des Herzens \\
\hline 1 & $\stackrel{g}{1260}$ & $\begin{array}{c}\mathrm{cg} \\
254\end{array}$ & $\begin{array}{c}\operatorname{cg} \\
202\end{array}$ & 3,44 & $\begin{array}{c}\text { Tage } \\
11\end{array}$ & $\begin{array}{l}\text { Rundes Loch auf einer. Aortenklappe. } \\
\text { Dilatation des linken Ventrikels. }\end{array}$ \\
\hline 2 & 900 & 270 & 300 & 3,02 & 12 & $\begin{array}{l}\text { Grosse Spalte auf einer Aortenklappe. } \\
\text { Dilatation des linken Ventrikels. }\end{array}$ \\
\hline 3 & 1380 & 389 & 282 & 1,63 & 19 & \\
\hline 4 & 780 & $366^{\circ}$ & 469 & 1,39 & 30 & \\
\hline 5 & 740 & 265 & 358 & 1,79 & 38 & Gr. Spalte aut einer Aortenklappe. Dila- \\
\hline 6 & 1220 & 444 & 364 & 1,47 & 42 & \\
\hline 7 & 810 & 303 & 374 & 1,77 & 46 & \\
\hline 8 & 870 & 373 & 4.29 & 1,98 & 46 & $\begin{array}{l}\text { Eine Aortenk lappezusammengeschrumpft. } \\
\text { Dilatation u. Hypertr. des l. Ventrikels. }\end{array}$ \\
\hline 9 & 2200 & 912 & 415 & 1,33 & 85 & \\
\hline 10 & 880 & 300 & 341 & 2,05 & 88 & Sectionshefund wie bei $\mathrm{No} 3-7$ \\
\hline 11. & 1340 & 638 & 476 & 1,14 & 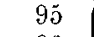 & \\
\hline 12 & 910 & 458 & 503 & & 98 & \\
\hline 13 & 840 & 288 & 324 & 2,24 & 122 & $\begin{array}{l}\text { Auf einer Aortenklappe ein kleines run- } \\
\text { des Loch mit rerdickten Rändern. Di- } \\
\text { latation und Hypertr. des 1. Ventrikels. }\end{array}$ \\
\hline 14 & 1380 & 454 & 329 & 1,59 & 126 & Wie bei No. $9-12$ \\
\hline 15 & 1170 & 658 & 562 & 1,03 & 155 & $\begin{array}{l}\text { Auf einer Aortenklappe eine Spalte und } \\
\text { eine Klappe ganz geschrumpft. Dila- } \\
\text { tation u. Hypertrophie des l. Ventrikels. } \\
\text { Wie bei No. 14. }\end{array}$ \\
\hline
\end{tabular}

Zur Constatirung der Hypertrophie habe ich das Herzgewicht, bezw. das relative Herzgewicht: $R$ ( $=$ Herzgewicht auf $1000 \mathrm{~g}$ Körpergewicht berechnet) und $\mathrm{J}(=$ Verhältnisszahl zwischen Nieren und Herzgewicht) verwendet. Warum ich diese Verhältnisszahlen gewählt habe, habe ich schon in Capitel II motivirt. In Tabelle $V$ sind diese Gewichtsverhältnisse mit den anderen auf die Hypertrophie bezüglichen Daten zusammengestellt.

Hypertrophie musste ich in jedem Falle annehmen, wenn $R$ den normalen Mittelwerth des relativen Herzgewichtes $257,9 \mathrm{cg}$ bedeutend überschritt und $\mathrm{J}$ wesentlich kleiner war als 2,82. War der Contrast zwischen diesen beiden Verhältnisszahlen ein sehr grosser, ohne dass auch nur eine die äussersten normalen Grenzen überschritten hätte, habe ich die entsprechenden Herzen nicht als hypertrophische ansprechen können. So konnte z. B. 
bei Fall 2 in Tab. $V$ noch keine Herzhypertrophie angenommen werden, trotzdem $R=300 \mathrm{cg}$, also bedeutend über den normalen Mittelwerth hinaus war, weil J nur 3,02, also noch bedeutend grösser als sein normaler Mittelwerth 2,82 war. Hingegen war im Falle 3 das Herz entschieden hypertrophisch, obwohl $R=282 \mathrm{eg}$, also noch innerhalb der maximalen normalen Grenze war, weil $J=1,63$ ist, welcher Werth bei normalen Herzen nie vorkommt.

Das absolute Gewicht des grössten hypertrophischen Kaninchenherzens war $912 \mathrm{cg}$ (Fall 9), also bedeutend grösser als das des grössten normalen Kaninchenherzens. Es wäre jedoch irrthümlich zu glauben, dass dieses Herz auch das im höchsten Grade hypertrophische war, da doch den Grad der Hypertrophie die Verhältnisszahlen $R$ und $J$ ausdrücken. $R$ war aber in jenem Falle 9 nur $415 \mathrm{cg}$, während bei Fall 15 es $562 \mathrm{cg}$ betrug; die Hypertrophie ist also grösser, trotzdem das absolute Herzgewicht nur $658 \mathrm{cg}$ war.

Der Grad der Hypertropbie hängt, soweit ich es aus meinen Experimenten bestimmen konnte, ab: 1. von der Zeidauer der Insufficienz, 2. von der Grösse des Klappendefectes. Ad 1) mögen die Fälle 3, 6, 11, 15 u. s. w., ad 2) die Fälle 11 und 13 als Beispiele dienen. Während im Falle 11 auf der defecten Klappe eine grosse Spalte war, fand sich bei Fall 13 nur ein kleines rundes Loch mit etwas verdickten Rändern auf derselben und deshalb entwickelte sich bei ersterem in 95 Tagen eine grössere Hypertrophie, als bei letzterem in 122 Tagen. Ausserdem hängt der Grad der Hypertrophie 3. vom Ernährungszustand des Herzens ab, auf den man aber nicht ohne Weiteres aus dem allgemeinen Ernährungszustand des ganzen Organismus folgern darf. Eben diesen Punkt will ich besonders hervorheben. Viele meiner Versuchsthiere sind während der Beobachtung nach der Operation sehr abgemagert, ja einige in solchem Maasse, dass sich die Hypertrophie unter den ungünstigsten Ernährungsverhältnissen entwickeln musste. Sie entwickelte sich trotzdem und es scheint, sie kann sich so lange entwickeln, bis im Herzen selbst Ernährungsstörungen auftreten. Am besten kann diese Behauptung mit Fall 15 unterstützt werden. Das Körpergewicht dieses Thieres sank während der 155 tägigen Beobachtungsdaner 
von $1179 \mathrm{~g}$ auf $840 \mathrm{~g}$ und $z$ war allmählich, wie das wiederbolte Messungen gezeigt haben. Doch hat sich bei diesem Kaninchen der höchste Grad der Hypertrophie entwickelt. Ich glaube also aus diesen Beobachtungen mit Recht folgern zu können, dass sich die Herzhypertrophie auch bei sehr ungüstigem allgemeinem Ernährungszustande entwickeln kann ${ }^{1}$ ). Diesem experimentellen Resultate widersprechen auch die klinischen . Erfahrungen nicht.

Bei hochgradiger Hypertrophie und Dilatation waren auch die Papillarmuskeln im linken Ventrikel verändert. Sie waren verflacht, ihr oberster Theil war zu einem sehnigen Gebilde umgewandelt, dass sich in die verlängerten Chordae tendineae fortsetzte. Solche atrophische Papillarmuskeln zeigten schon makroskopisch meistens hochgradige fettige Degeneration. Diese Befunde gewinnen dadurch an Interesse, weil kein einziges Symptom im Leben darauf hinwies, dass durch diese Veränderungen der Papillarmuskeln die Schliessung der Mitralklappe eine unvollkommene geworden wäre. Es ist kein $Z$ weifel darïber, dass die Ursache dieser Veränderung der Papillarmuskeln die grosse Ausdehnung derselben während der Ventrikeldilatation ist.

Schliesslich sei noch ein nicht uninteressanter Umstand erwähnt. Bei allen meinen herzkrank gemachten Versuchsthieren hat der hypertrophirende Ventrikel so vollkommen der grösseren Arbeitsanforderung entsprochen, dass sich Symptome der Incompensation nicht gezeigt haben. Nur in einem einzigen Falle fand ich am 1õ5. Tage der Insufficienz bei der Section einen nicht grossen Hydrops ascites. Die Thiere, die ich nicht selbst tödtete, starben ohne irgend ein Zeichen geschwächter Herzfunction - und oft ohne dass die Section eine Todesursache bätte constatiren können. Was die Ursache dieser Verschiedenheit zwischen den künstlichen und pathologischen Herzfehlern ist, konnte ich nicht sicher ermitteln. Es scheint mir jedoch als ob

1) Einer irrthümlichen Deutung der Daten in Tab. V, S. 331 vorzubeugen, sei es hier erwähnt, dass zur Bestimmung des relativen Herzgewichtes immer das zur Zeit der Operation beobacbtete Körpergewicht herangezogen wurde, und nie das bei der Section gefundene. Das musste eben mit Rücksicbt auf die beohachtete Abmagerung der Thiere gescbehen. 
die gestörte Function der Herzmusculatur -- die unmittelbare Ursache der Incompensation - erst durch andere Veränderungen im Organismus, vielleicht in den meisten Fällen durch die des Blutgefässsystems bedingt, bezw. hervorgerufen wird. Diese Veränderungen fehlten bei den künstlichen Herzfehlern. Natürlich will ich damit nur eiue Vermuthug ausgesprochen haben.

Die histologische Cntersuchung der hypertrophischen Herzen erstreckte sich in erster Reihe auf die Feststellung des Wesens dieses pathologischen Wachsthumes, also ob die Hypertrophie auf der Vergrösserung oder Vermehrung dér Muskelzellen oder auf beiden beruht. Es mussten deshalb dieselben Messungen vorgenommen werden, wie bei den normalen Herzen, ich muss also hier nicht die Beschreibung der Messungsmethoden wiederholen. Der Mittelwerth des Muskelzellenquerdurchmessers ist in jedem Falle aus 100 Einzelmessungen berechnet. Diese Mittelzahlen finden sich in Tabelle VI.

Hier sind im Ganzen nur 13 Fälle aufgezäht. Die Fälle 1 und 2 (s. Tabelle V, S. 331) mussten weggelassen werden, weil ich diese Herzen aus früher angegebenen Gründen nicht für hypertrophisch halte. Fall 8 blieb weg, weil ich wegen dazwischen gekommenen Hindernissen nur 50 Zellen messen konnte und so die aus dieser gewonnene Mittelzahl unter die anderen als gleichwerthige nicht aufgenommen werden kounte.

Tabelle VI.

Nuskelzellenquerdurchmesser hypertrophischer

Kaninchenherzen.

$\mathrm{D}=$ Mittelwerth des Querdurchmessers aus je 100 Messungen berechnet.

\begin{tabular}{c|c|c|c|c|c}
\hline $\begin{array}{c}\text { No. nach } \\
\text { Tab. V. }\end{array}$ & $\begin{array}{c}\text { Abs. } \\
\text { Herzgewicht }\end{array}$ & $\mathrm{D}$ & $\begin{array}{c}\text { No. nach } \\
\text { Tab. V. }\end{array}$ & $\begin{array}{c}\text { Abs. } \\
\text { Herzgewicht }\end{array}$ & $\mathrm{D}$ \\
\hline 5 & $265 \mathrm{cg}$ & $13,42 \mu$ & 14 & $454 \mathrm{cg}$ & $16,42 \mu$ \\
13 & $288-$ & $14,20-$ & 12 & $458-$ & $16,25-$ \\
10 & $300-$ & $15,19-$ & 11 & $638-$ & $17,63-$ \\
7 & $303-$ & $14,14-$ & 15 & $658-$ & $17,37-$ \\
4 & $366-$ & $14,70-$ & 16 & $712-$ & $20,97-$ \\
3 & $389-$ & $14,76-$ & 9 & $912-$ & $20,17-$ \\
6 & $444-$ & $15,50-$ & & &
\end{tabular}

Wie bei den normalen Herzen habe ich auch hier die Daten der Tabelle VI in der folgenden Tabelle VII graphisch dargestellt um sie übersichtlicher zu veranschaulichen. 
Tabelle VII.

Vergrösserung des Muskelzellenquerdurchmessers bei der Hypertrophie des Herzens.

D $=$ Mittelwerth des Querdurchmessers aus je 100 Messungen berechnet.

$D^{\prime}=$ Gesammtmittel einer Gruppe aus den D derselben berechnet.

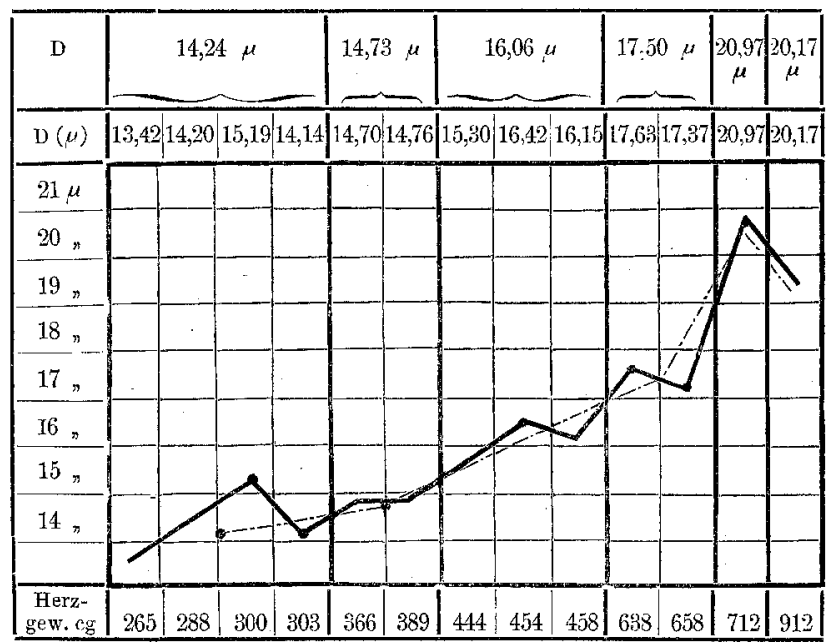

Hier sind nach demselben Principe Gruppen gebildet and ans den D einer Gruppe ein Gesammtmittelwerth berechnet, dessen Verhalten die punctirte Curve versinnlicht. Die ausgezogene Curve bezieht sich auf $D$.

Auf dieser Tabelle sehen wir ähnliche Verhältnisse ausgedrückt, wie bei den normalen Herzen, je grösser das absolute Herzgewicht, desto grösser der Muskelzellenquerdurchmesser, d. h. wie bei dem physiologischen, wird auch bei dem pathologischen Wachsthum, bei der Hypertrophie des Herzens mit dem Wachsen des absoluten Herzgewichtes der Querdurchmesser der Muskelzellen proportional grösser.

Die Verlässlichkeit der gewonnenen Mittelwerthe wurde hier auch geprüft. Tabelle VIII liefert die Daten dieser mathematischen Untersuchung. 
Tabelle VIII.

$\mathrm{D}=$ Mittelwerth des Muskelzelle nquerdurchmessers aus je 100 Vessungen bestimmt;

$\mathrm{W}=$ wahrscheinlicher Werth der Abweichung der einzelnen Messungen vom Mittelwerthe I)

$F=$ wahrscheinlicher Werth des Bestimwungsfehlers der Mittelzahl D.

(Im Theile 2: Häufigkeit und Grössenwerth der einzelnen Abweichungen von dem Mittelwerthe D.)

\begin{tabular}{|c|c|c|c|c|c|c|c|c|c|c|c|c|c|c|c|}
\hline \multicolumn{5}{|c|}{1.} & \multicolumn{11}{|c|}{2.} \\
\hline 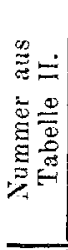 & $\begin{array}{c}\text { Abs. } \\
\text { Herz- } \\
\text { gewicht }\end{array}$ & D) & $\begin{array}{l}\text { W } \\
a\end{array}$ & $\mu$ & 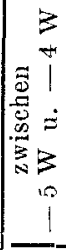 & 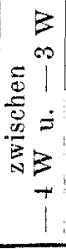 & 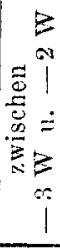 & 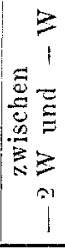 & 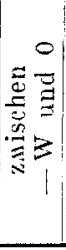 & $\begin{array}{l}\frac{3}{5} \\
\frac{D}{0}+ \\
0 \\
0 \\
0 \\
0 \\
0\end{array}$ & 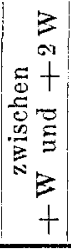 & $\begin{array}{r}3 \\
0 \\
0+1 \\
0 \\
0 \\
0 \\
02 \\
0 \\
+1\end{array}$ & 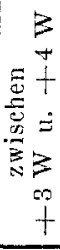 & 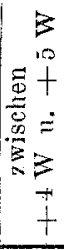 & 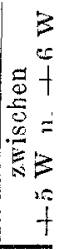 \\
\hline 5 & $35 \mathrm{cg}$ & 42 & 1,96 & 0 & 0 & 0 & 10 & 2 & 31 & 31 & 16 & 6 & 3 & 1 & \\
\hline 10 & $300-$ & 10,19 & 2,29 & 0,2 & 0 & 1 & 5 & 16 & 33 & 26 & 11 & 4 & 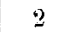 & 1 & \\
\hline 4 & $366-$ & 14,70 & 1,98 & 0,2 & 0 & 0 & s & 18 & 19 & 36 & 16 & 0 & & 0 & 1 \\
\hline 3 & $389-$ &, 76 & $-2,03$ & 0,2 & 0 & c & 5 & 20 & 33 & 34 & 11 & 3 & & 4 & 0 \\
\hline 6 & $444-$ & 15,50 & 1,94 & 0 , & 0 & 3 & 0 & 11 & 34 & 38 & 4 & 6 & & 0 & 0 \\
\hline 14 & 454 & 16 & 2,44 & & 0 & 1 & 13 & 21 & 29 & 21 & 8 & 4 & & & 0 \\
\hline 11 & 63 & 17 & 2,40 & 0 & () & 5 & 5 & 20 & 21 & 28 & 14 & 8 & & & 1 \\
\hline 15 & $658-$ & 17,37 & 2,90 & & 0 & & 4 & 20 & 36 & 19 & 11 & & & & I \\
\hline $16^{\circ}$ & $712-$ & & 2,69 & & c & 0 & 7 & 11 & $4 !$ & 28 & 6 & & & 4 & 0 \\
\hline 9 & $912-$ & 20,17 & 2,51 & 0,25 & 1 & 0 & 4 & 19 & 23 & 28 & 14 & 6 & 4 & 1 & 0 \\
\hline & & & & & 1 & 10 & 61 & 158 & 300 & $\because 74$ & 111 & & & 18 & 4 \\
\hline & & & DI & & 4 & 18 & 67 & 161 & 250 & 250 & $16 !$ & 67 & 18 & 4 & 0 \\
\hline - & - & $-\quad-$ & 100 & & 1 & 2 & 6 & 16 & 25 & 25 & 16 & 6 & 2 & 1 & - \\
\hline
\end{tabular}

Aus dieser Tabelle geht zur Genïge hervor, dass diese Nittelwerthe dieselben Verhältnisse aufweisen, wie die bei den normalen Herzen gewonnenen. Die Grösse der Bestimmungsfehler ist auch hier weit innerhalb der erlaubten Grenzen und die Asymmetrien in den Reihen der Häufigkeitszahlen stimmen auch mit denen in Tabelle IV S. 326 überein; nur kommen hier in vier Beobachtungsreihen je eine Abweichung vor, die zwischen $+5 \mathrm{~W}$ und $+6 \mathrm{~W}$ stehen. Die Erklärung und Bedeutung dieser Asymmetrien und Unregelmässigkeiten ist dieselbe, wie die der Tabelle IV. Wir müssen, wie bei den normalen, so auch bei den hypertrophischen Herzen mit Thoma annehmen, dass die gemessenen, pathologisch gewachsenen Zellen nicht gleichwerthig sind. Diese Analogie zwischen normalen und hypertrophischen Herzen weist, auch daranf hin, dass das Wachsthum beider auf denselben Factoren berult. 
Das Dickerwerden der Muskelzellen wäre nun festgestellt doch wächst bei der Hypertrophie auch der Längendurchmesser der Zellen. Ueber methodische Messungen verfüge ich auch hier nicht, doch kann ich aus den wenigen Messungen, die ich angestellt habe und aus den sichtlichen Differenzen der Länge zwischen Muskelzellen aus kleineren und grösseren hypertrophischen Herzen, entschieden folgern, dass gleichzeitig mit dem Dickenwachsthume die Muskelzellen auch länger werden. Dass in sehr grossen hypertrophischen Herzen, z. B. in dem mit $912 \mathrm{cg}$, in den zwei kernigen Zellen die Kerne gewöhulich weiter von einander stehen, spricht auch dafür. Ausserdem zeigt auch die Gestaltverïnderung des Ventrikels - wie das schon Goldenberg betont hat - dass die Muskelfaserbündel and somit auch die Muskelzellen sich verlängern müssen. Ob aber das Jü̈ngenwachsthum der Zellen mit dem Dickenwachsthum gleichen Schritt hält, ist sehr fraglich. Es scheint so, als ob das nicht der Fall wäre. Sicher werden das erst vielleicht spätere, auf diesen Punkt gerichtete Messungen entscheiden; jetzt lässt es sich nur vermuthen.

Bei Vergleichung der Mittelwerthe von Muskelzellenquerdurchmessern normaler und hypertrophischer Herzen stellt es sich heraus, dass bei Herzen mit gleichem (absoluten) Gewichte die Muskelzellen des hypertrophischen fast in allen Fällen grösser sind als die des normalen. So ist $z$. B. im $265 \mathrm{cg}$ schweren normalen Herzen (Tab. II, Fall 6) $\mathrm{D}=11,21 \mu$, während bei einem ebenso schweren hypertrophischen (Tab. VI, Fall 5) $\mathrm{D}=13,41 \mu$ ist. Aebnlich verhalten sich auch die anderen Fälle. Wollen wir jedoch aus diesem Unterschiede irgend eine Folgerung ziehen, müssen wir bedenken, dass erstens dieser Unterschied ein zufälliger, individueller sein kann, da auch bei fast gleich schweren Herzen die Mittelwerthe um $1-2 \mu$ variiren können. Zweitens dürfen wir nicht ausser Acht lassen, dass der Werth dieses Unterschiedes in einigen Fällen innerhalb der Grenzen des Bestimmungsfehlers des Mittelwerthes liegt. Doch scheint der Umstand, dass dieser Unterschied constant fast bei jedem Falle zu beobachten ist, es wahrscheinlich zu machen, dass dieser Unterschied kein zufälliger ist. Dieser würde dann darauf hinweisen, dass bei der Hypertrophie der Volumsver- 
grösserung der Muskelzellen eine noch bedeutendere Rolle zufällt als beim physiologischen Wachsthume des Herzens.

Neben der nun erwiesenen Volumsvergrösserung der Muskelzellen musste noch besonders auf ihre eventuelle numerische Vermehrung geachtet werden. Die numerische Vermehrung der Muskelzellen ist auf zweierlei Art möglich: entweder durch Theilung der schon vorhandenen Muskelzellen oder durch Metaplasie anderer Gewebsbestandtheile im Muskelgewebe. Letulle ${ }^{1}$ ) meint, dass, die Vermehrung der Muskelfasern vorausgesetzt von der er sich übrigens nie überzeagen konnte -, die schon vorhandenen Muskelfasern dabei gar nicht betheiligt wären, sondern dass eine Neubildung aus Bindegewebszellen vor sich gehen würde. Rindfleisch ${ }^{2}$ ) und $W_{e i s m a n n^{3}}$ ) sind der Ansicht, dass beim Wachsen der quergestreiften Musculatur eine Theilung der Muskelfasern stattfindet. Rindfleisch fand in hypertrophischen Herzen Muskelzellen mit zwei oder mehr Kernen, so wie sie Weismann in Muskelfasem fand, die seiner Meinung nach in Theilung begriffen waren, die mit Spaltenbildung beginnen soll. Auch Goldenberg glaubt, dass sich einige Fasern der Lünge nach theilen.

Vor Allem fand ich nie solche, aus denen man auf irgend eine Art vermuthen hätte könneu, dass sie Uebergangsformen seien zwischen Bindegewebs- und Muskelzellen. Ich sah keine Spur einer Muskelzellenbildung im Bindegewebe; auch ist es noch sehr fraglich, ob überhaupt aus Bindegewebe Muskelfasern entstehen können.

Was die Kerne der Muskelzellen betrifft, traf ich weder in hypertrophischen, woch in normalen Herzen solche, die inehr als zwei Kerne enthielten, so dass mehr kernige Zellen überhaupt nicht (oder nur in einer sehr kleinen Zahl) vorhanden sein müssen. Auch könnte ich nicht sagen, dass in der hypertrophischen Herzmusculatur die zweikernigen Zellen in grösserer Zahl wären als in der normalen. Somit entfiele schon das eine Symptom der Zellentheilung. Trotz des eifrigsten und aufmerksamsten Suchens ist es mir nicht gelungen, in den zahlreichen Prä-

1) Letulle, a. a. O. S. 90.

3) Rindfleisch, Lehrb. d. path. Gewebelehre. 6. Aufl. S. 236.

3) Weismann, Ueber d. Wachs. ete, Zeitschr f. rat. Med. Bd. X. S. 263. 
paraten aus hypertrophischen Herzen, die ich genau nach Flemming's Vorschriften hergestellt habe, in den Muskelzellen Karyomitosen $z u$ finden, ebenso wenig, wie in entwickelteren normalen Herzen: Wenn auch diesem negativen Befunde keine entscheidende Beweiskraft zukommt ${ }^{1}$ ), muss ich mich auf Grund derselben doch Goldenberg und Letulle anschliessen, die auch behaupten, dass bei der Hypertrophie des Herzens die centralen Kerne der Muskelzellen sich nicht vermehren.

Wie steht es nun aber mit den übrigen Zeichen der Zelltheilung? Die von Goldenberg angegebenen Befunde können meiner Meinung nach nicht die Theilung der Muskelzellen beweisen. Goldenberg beschreibt Fasern, die auf dem Querschnitte keine einfache rundliche Gestalt besitzen, sondern gelappt sind, einspringende Winkel darbieten, als "seien sie aus. mehreren dicht neben einander gruppirten Muskelzellen aufgebaut". In einigen Fällen ist es ihm auch gelungen, die Contouren dieser einzelnen Zellen aufzufinden. Goldenberg sagt übrigens, dass diese "Bündelfasern", wie er sie nennt, in den hypertrophischen Herzen nur etwas häufiger sind, als in normalen. Aehnliche Fasern fand ich auch in meinen Präparaten, doch glaube ich aus der gelappten Gestalt allein noch nicht auf Theilung folgern zu dürfen, selbst dann nicht, wenn man die Contouren der einzelnen aus Theilung hervorgegangenen Zellen zu sehen meint. Die Muskelzellen sind nehmlich verzweigt, auch in 2-3 $Z_{w}$ eige, die oft nicht einmal zur Hälfte so breit sind, wie der unverzweigte Theil der Zelle. Trifft nun der Querschnitt die Zelle an der Verzweigungsstelle oder in unmittelbarer Nähe derselben, so muss er naturgemäss gelappt erscheinen mit einspringenden Winkeln and die sehbaren Contunren der vermeintlichen aneinander gruppirten Zellen entsprechen denen der einzelnen Aeste. - Zweitens müsste man, wenn diese „Bündelfasern" durch Längstheilung einer Zelle entstanden wären, bei der Isolirung der Muskelzellen in Kalilauge, viele kernlose Zellen finden, da auch Goldenberg selbst zugiebt, dass trotz der Zelltheilung die Muskelkerne sich nicht vermehren. Solche kernlose vollständige Zellen konnte ich nicht auffinden, denn kernlose,

1) Es wäre ja nicht unmöglich, dass Mitosen nur in sehr geringer Zahl oder nur periodenweise in einigen Zellen auftreten. 
durch die Präparirung entstandene Bruchstücke einzelner Zellen konnte ich doch nicht für kernlose Zellen halten. Wohl habe auch ich sowohl in normalen als hypertrophischen Herzen bei der Isolirung der Maskelzellen welche gefunden, in denen die Spalte zwischen den Zellästen so tief in den Zellkörper einschnitt, dass derselbe fast in zweien getheilt erschien. Auch sah ich in einigen Zellen eine Spalte mitten im Zellkörper, ohne dass dieselbe die Enden der Zelle erreicht hätte. Also eine Spalte in einer unverzweigten Zelle. (Rindfleisch meint, dass solche Spaiten später sich vergrössern und verbreitern und auf diese Art entwickelte sich dann ein neuer Ast des Muskelbalkenwerkes.) Diese Zellen enthielten aber nicht immer 2 Kerne.

Allerdings können das Erscheinungen sein, die vielleicht auf eine Theilung der Zellen hinweisen, doch glaube ich entschieden, dass man aus diesen allein noch nicht auf eine Theilung folgern kann. Dafür müssten andere sichere Zeichen vorhanden sein. Da ich nun über gar keinen Befund verfüge, der die Vermehrung der Muskelzellen beweisen würde, scheint es mir wahrscheinlicher zu sein, dass sie äberhaupt nicht stattfindet. Wie dem aber auch sei, würde auch der eventuellen Vermehrung der Zellen bei der Hypertrophie des Herzens nur eine ganz nebensächliche Rolle zufallen.

Die Ergebnisse meiner Untersuchungen führen also zu dem Schlusse, dass das Wesen der Hypertrophie des Herzens in der Vergrösserung der Muskelzellen besteht, deren prägnantester Ausdruck das Messungsresultat ist, nach welchem mit dem Herzgewicht der Mittelwerth der Muskelzellenquerdurchmesser gleichmässig wächst.

Zum Schlusse muss ich über die übrigen histologischen Vorgänge in der hypertrophischen Herzmusculatur Einiges mittheilen. Was die Muskelzellen betrifft, so sind die Kerne derselben, wie im normalen Herzen, von sehr verschiedener Gestalt, meist oval; oft scheinen sie in ihrer Mitte eine Einschnürung zu haben; oft sind sie sehr lang und überhaupt scheinen sie sich mit der Zelle auch zu vergrössern. Mitosen fand ich nie. Die verschiedenen Einkerbungen, die man an ihnen in Schnitten sieht, glaube ich grösstentheils auf die Wirkung der angewendeten Reagentien zurückführen zu können, und nicht, wie Letulle, auf den Druck der hypertrophirenden Muskelfibrillen. 
Um den Kern herum ist der Körper der Muskelzelle gewöhnlich mehr uder minder verändert. Der Kern erscheint dann von einem aus einer körnigen Substanz gebildeten Hof umgeben, welcher allmählich in den peripherischen Theil der Zelle übergeht. Die glänzenden Körner dieser Substanz sind beiläufig so gross, wie die Quersclnitte der Muskelfibrillen, habeu ähnlichen Glanz und verhalten sich gegen Reagentien wie diese. Sie sind entschieden keine Fettkörner, sie färben sich mit Osmiunsäure nicht schwarz und geben auch die übrigen Fettreactionen nicht, wovon man sich am besten bei Zellen überzengen kann, in denen wirkliche Fettkörnchen vorhanden sind. Die Bedeutang dieser Körner, ihre Natur und ihr Verhältniss zur quergestreiften Substanz konnte ich nicht feststellen. Möglicberweise stehen sie mit dem Wachsthum der Zellen in irgend einem Zusammenhange. Die Vermehrung dieser Körner scheint mit der Vermehrung des sogenannten "Protoplasma" um den Zellkern herum vor sich zu gehen. Dieses "Protoplasma" scheint sich aber bei der Hypertrophie nicht in allen Zellen zu vermehren; in vielen Zellen findet man es aber weit über die Pole des Kernes hinaus noch vermehrt.

Oft genug fand ich eine mehr oder minder verbreitete fettige Degeneration in den Muskelzellen, doch habe ich zwischen dieser Degeneration und der Zeitdauer der Hypertrophie keinen Zusammenhang constatiren können, so dass ich mit Letulle ganz und gar nicht einverstanden bin, der zwei Stadien der Hypertrophie unterscheidet, das der „Hypernutrition“ und das der Degeneration, wo die letztere die nothwendige Folge der ersteren wäre.

Ausserdem sah ich noch in einigen Zellen kleine runde Höhlen, kleine Vacuolen. Es ist nicht unmöglich, dass diese in der saftreicher gewordenen Zellensubstanz erst unter dem Einfluss der Fixirungsflüssigkeit entstehen, etwa so wie die Vacuolen in dem durch Flemming'sche Flüssigkeit fixirten geronnenen Blute.

Was die Veränderungen der übrigen histologischen Bestandtheile der Herzwand betrifft, so verfüge ich nur bezüglich des Bindegewebes über einige Befunde. Es ist allgemein bekannt, dass bei der Herzhypertrophie gleichzeitig mit der Muskelsubstanz auch das Bindegewebe sich vermehrt und so zur Volumsvergrösserung des Herzens beiträgt. Letulle meint sogar, dass es die Vermehrung des Bindegewebes sei, welche in Folge der durch 
sie bedingten Ernährungsstörung die - nach Letulle - bei jeder Hypertrophie eintretende Jegeneration der Muskelfasern hervorrufe. Von dieser wichtigen Rolle des Bindegewebes konnte ich mich nicht überzeugen. Ich fand in fiühen Stadien der Hypertrophie nie Vermehrung des Bindegewebes, so dass ich berechtigt bin zu behaupten, dass mit der Vergrösserung der Muskelzellen gleichzeitig eine Vermehrnng des Bindegewebes nicht stattfindet. Auch in den späteren Stadien fand ich es nicht immer vermehrt, und wo es vermehrt war, nicht in hohem Grade. Am häufigsten schienen jene Balken verdickt, die sich aus dem Pericard in die Muskelsubstanz fortsetzen. Aus diesen Balken erstreckte sich in einigen Fällen eine kleine Bindegewebswucherung auf kurze Strecken zwischen die Muskelfaserbündel hinein. Einigemal sah ich anch um die einzelnen Muskelzellen ein aus feinen, lockeren, viele Lücken, wahrscheinlich erweiterte lymphräume zwischen sich schliessenden Fibrillen bestehendes Bindegewebe. Nie war aber das Bindegewebe diffus durch die ganze Herzwand vermehrt und verdickt. (In einigen Herzen war unmittelbar unter dem Endocard an einigen umschriebenen Stellen - die makroskopisch wie kleive Sehnenflecke aussahen - eine Bindegewebswucherung, in welcher atrophische und degenerirte Muskelzellen eingeschlossen waren. Diese Stellen entsprachen wahrscheinlich jenen Verletzungen, die bei der Operation durch die in den Ventrikel gelangte Sonde verursacht wurden.) Ich glaube also überhaupt nicht, dass der Bindegewebsvermehrung bei der Volumsvergrösserung des Herzens eine wesentliche Rolle zukommt. Auch gehört sie nicht zum Wesen der Herzhypertrophie, und dass sie im hypertrophischen Herzen des Menschen so oft angetroffen wird, ist meiner Meinung nach so za erklären, dass hier die Bindegewebswucherung in directem Zusammenhange steht mit jenen entzünlichen Prozessen, welche in der Intima der Arterien oder im Endocard des hypertrophischen Herzens vorangehen. Bei den künstlichen Herzfehlern der Versuchsthiere fehlten jedoch diese entzündlichen Prozesse, deshalb blieb auch die Bindegewebswucherung aus, die also nicht nothwendigerweise die Vergrösserung der Muskelzellen begleiten muss. Letztere bleibt in allen Fällen das wesentliche Moment der Herzvergrösserung. 